\title{
A clinical trial of supervised exercise for adult inpatients with acute myeloid leukemia (AML) undergoing induction chemotherapy
}

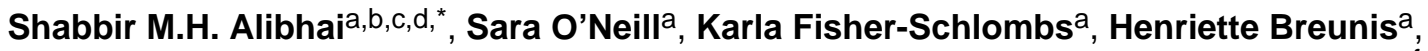 \\ Joseph M. Brandwein ${ }^{a, c}$, Narhari Timilshina ${ }^{a}$, George A. Tomlinson ${ }^{a, e}$, Heidi D. Klepin ${ }^{\dagger}$, and \\ S. Nicole Culos-Reedg,h \\ aDepartment of Medicine, University Health Network, Toronto, ON, Canada \\ ${ }^{b}$ Geriatric Program, Toronto Rehabilitation Institute, Toronto, ON, Canada \\ 'Department of Medicine, University of Toronto, Toronto, ON, Canada \\ dInstitute of Health Policy, Management and Evaluation, University of Toronto, Toronto, ON, \\ Canada \\ eDepartment of Public Health Sciences, University of Toronto, Toronto, ON, Canada \\ fDepartment of Hematology and Oncology, Wake Forest University, Winston-Salem, NC, USA \\ gDepartment of Kinesiology, University of Calgary, Calgary, AB, Canada \\ hDepartment of Psychosocial Resources, Tom Baker Cancer Centre, Calgary, AB, Canada
}

\begin{abstract}
Patients with acute myeloid leukemia (AML) receiving induction chemotherapy (IC) were enrolled in a supervised exercise intervention to determine safety, feasibility, and efficacy. Physical fitness measures, quality of life (QOL) and fatigue were assessed using standardized measures at baseline, post-induction, and post first consolidation. Retention was excellent, the intervention was safe, and efficacy estimates suggested benefits in physical fitness and QOL outcomes. Exercise is a safe, promising intervention for improving fitness and QOL in this patient population. These results provide a foundation for a randomized trial to better understand the impact of exercise during IC on clinically important outcomes.
\end{abstract}

\section{Keywords}

Acute myeloid leukemia; Chemotherapy; Exercise; Pilot study; Quality of life; Cancer-related fatigue; Elderly; Physical fitness

\footnotetext{
Clinical Trial Registration Number: NCT01170598.

(C) 2012 Elsevier Ltd. All rights reserved.

"Corresponding author at: University Health Network, 200 Elizabeth Street Room EN14-214, M5G 2C4, Toronto, ON, Canada. Tel.: +1 416340 5125; fax: +1 416595 5826. shabbir.alibhai@uhn.on.ca (S.M.H. Alibhai).

Contributions. SA was responsible for the conception, design and implementation of the study, on-going study support and drafting of the manuscript. SO, KF and HB were responsible for patient recruitment and administration of the intervention and assessments. JB, HK and NCR provided advice on protocol development, study implementation and patient safety. NT and GT were responsible for data analysis and interpretation. All authors contributed to the writing of the manuscript and approved the final version.
}

Conflict of interest

None of the authors have any financial or personal conflicts of interest to declare. 


\section{Introduction}

Acute myeloid leukemia (AML) is a life-threatening malignant blood disorder that primarily affects older adults [1]. Curative treatment requires multiple cycles of intensive chemotherapy. The first cycle, induction (IC), is the most intense, requires 4-6 weeks of inpatient admission, and is associated with extended bed rest and multiple toxicities, leading to physical deconditioning. Treatment-related mortality with IC is significant, ranging from 8 to $15 \%$ in older adults $[2,3]$. Achieving complete remission (CR) typically

AML and its treatment with intensive chemotherapy are both associated with significant negative impacts on quality of life (QOL) [4-6]. In particular, investigators have found that QOL and self-reported physical function are lowest three weeks after each course of chemotherapy [4-7]. Fatigue has been found to be one of the most common [5,6,8] and distressing $[4,5]$ symptoms, and is reported by over $90 \%$ of AML patients during treatment [7].

Reductions in aerobic fitness, muscular strength, and endurance have been observed during chemotherapy for a variety of malignancies, although data in AML are limited [9-13]. Declines in fitness and strength not only contribute to difficulty with activities of daily living but also worsen fatigue and may reduce the ability to tolerate further chemotherapy or lead to delays in chemotherapy administration [9-12].

Exercise during chemotherapy can significantly ameliorate these effects $[14,15]$. Randomized controlled trials (RCTs) of exercise in other cancer populations have demonstrated improvements in QOL, fatigue, aerobic capacity and muscle strength [15-17]. In addition, exercise may also reduce anxiety, depression, and distress [16,17].

Four previous studies have examined the feasibility and effects of exercise in adult patients with AML undergoing IC [18-21]. Study interventions consisted of exercise regimens 3-5 days per week for up to 45 min per day, with exercises ranging from walking to mixedmodality programs. In general, all 4 studies found improvements in patient-reported QOL (including fatigue, depression, and distress) and fitness (aerobic capacity, endurance, and strength) outcomes. However, all 4 studies featured small sample sizes (10-24 patients) and included predominantly younger patients, whereas most AML patients are elderly. Older adults have less physiologic reserve, are less physically active, have greater comorbidities, and thus are likely to benefit more from exercise. However, they may also be able to tolerate it less. Finally, these studies provided limited formal data on safety and adverse events, which are crucial to the safe delivery of an exercise program [22].

Our research program is ultimately interested in improving QOL and treatment tolerability for all AML patients undergoing intensive chemotherapy. In this first phase, we designed a non-randomized pilot study to determine recruitment, retention, and ability of AML patients undergoing induction chemotherapy to participate in an individualized exercise intervention during hospitalization; to provide efficacy estimates on physical fitness outcome measures; and to examine the safety of the exercise program. Secondary objectives were to provide estimates of the effects of exercise on QOL and fatigue; and to understand the impact of exercise on AML treatment tolerability, including length of stay in hospital, development of sepsis, intensive care unit (ICU) admission, and delay in subsequent chemotherapy.

\section{Materials and methods}

\subsection{Study design}

This non-randomized clinical trial recruited patients between June 2010 and February 2011. All participants were stratified by age (under 60 and 60 or older) to allow us to investigate 
differences among younger and older patients' experiences and outcomes. All participants were recruited from Princess Margaret Hospital (PMH) in Toronto, Canada and provided written informed consent to participate. PMH is a tertiary care AML referral and treatment center. The study was approved by the institutional Research Ethics Board.

\subsection{Subject recruitment}

Patients were assessed for inclusion based on the following criteria: $\geq 18$-years-old; newly diagnosed AML or relapsed AML after having been in CR for at least 6 months; initiating IC; ambulatory without need for human assistance; fluent in English; and medically cleared for participation by the attending physician. Exclusion criteria included: another active malignancy; life expectancy < 1 month; significant medical comorbidity; uncontrolled pain; and hemodynamic instability. Subjects were enrolled within 5 days of starting chemotherapy. A log detailing reasons for exclusion and non-participation was kept for all approached patients.

\subsection{Intervention}

Each participant received an individualized, supervised, mixed-modality exercise program created by a Canadian Society for Exercise Physiology (CSEP)-Certified Exercise Physiologist (CEP). Following the baseline assessment, in which sociodemographic, clinical, QOL, and fitness information was obtained, each participant was approached 4-5 times per week to engage in light to moderate intensity exercise. Exercise sessions included a combination of aerobic exercise (walking or stationary cycling), resistance training (using resistance bands and/or free weights), and flexibility training (static stretching) for 30-45 min per session. The breakdown of each exercise session in terms of the time spent completing aerobic, resistance and flexibility training was not pre-established due to variability in exercise tolerance as well as disease and treatment related side-effects. We aimed to complete a minimum of $10 \mathrm{~min}$ of aerobic training (up to $40 \mathrm{~min}$ ) and $10 \mathrm{~min}$ of resistance training (up to $25 \mathrm{~min}$ ). Flexibility training was done at the end of each session and focused on the muscles exercised during that session, lasting 5-10 min.

Patients were given the opportunity to select either walking or cycling to complete the aerobic portion of each session. A choice was given so that participants had the opportunity to choose a mode of exercise that they preferred in the hope that this would enhance exercise adherence.

Aerobic exercise intensity was monitored using the rating of perceived exertion (RPE) scale [23] and heart rate monitors (Polar, NY, USA). Following an aerobic warm-up, patients were encouraged to exercise at an RPE of 3-6 (out of 10) or 50-75\% of their heart rate reserve as per American College of Sports Medicine (ACSM) guidelines [15].

As this was a pilot study, in the initial phase of the study we used both heart rate monitors and the RPE scale to monitor intensity throughout the exercise sessions. However, we found that it was obtrusive for patients to have to wear a heart rate monitor around their chest for each session due to intravenous lines. Since 50-75\% heart rate reserve (HRR) is equivalent to $3-6$ on the (0-10) RPE scale, we chose to use the RPE scale alone to monitor intensity. Regardless of the mode of exercise, walking or cycling, the speed or incline of the treadmill and the resistance on the bike was adjusted to elicit a response of 3-6 on the RPE scale.

Resistance exercises and flexibility training targeted large muscle groups. Resistance exercises varied among participants based on comfort level with different movements and familiarity with exercise and resistance training. Each participant completed exercises that targeted all of the major muscle groups, but the specific exercises completed varied among patients. The exercises that were used throughout the intervention are listed here. Leg 
exercises included: leg press, squat, lunge, calf raise, dead lift. Chest exercises included: wall push-up, chest press, single arm fly. Back exercises included: seated row, bent over row, pull down. Arm exercises included: biceps curl, hammer curl, triceps extension, triceps dip, triceps push-up. Shoulder exercises included: upright row, front raise, lateral raise, shoulder press. A selection of these exercises was completed each day. For example, 1-3 sets of leg, chest and back exercises were performed on day 1 and 1-3 sets of arm and shoulder exercises were performed on day 2 .

The design and execution of the exercise program did have to consider the intravenous lines and poles that each patient transports with them. As such, prior to executing each specific exercise, the CEP ensured that the movement would not interfere with the line. In addition, the line was positioned so as not to alter the range of motion of any particular exercise.

Exercise progression and adaptations were evaluated on a regular basis to ensure patient safety. Exercise progression for each patient varied depending on their baseline fitness, exercise tolerance as well as disease and treatment-related symptoms and side-effects. Ideally, both aerobic and resistance exercise progressed weekly as tolerated. Aerobic exercise was first increased in duration to achieve the desired length of exercise time (20-30 min). The following week the intensity of the exercise was increased (by either increasing the speed of walking, incline of the treadmill, or resistance of the bike). Resistance exercises were modified, also on a weekly basis by increasing the number of sets, repetitions or resistance. Again, this progression varied since some patients were able to work with a higher level of resistance, while others were simply able to increase the number of repetitions completed. Any change in exercise intensity was evaluated using the RPE scale to ensure that the exercise remained in the light to moderate range. Each patient received a personal package of resistance bands and all shared exercise equipment (i.e., treadmill) was carefully sanitized between each use to ensure appropriate infection control.

On each day of scheduled exercise, the CEP would speak with the nurse and the patient to ensure that there were no contraindications to exercise. Although target durations and intensities were selected, both varied based on patient tolerance, symptoms and blood parameters. If a patient's hemoglobin was $<80 \mathrm{~g} / \mathrm{L}$, the CEP would speak to both the patient and nurse about possible contraindications to exercise (e.g. lightheadedness), in which case the patient was approached after a transfusion was complete. If a patient's platelet count was $<10 \mathrm{bil} / \mathrm{L}$, the CEP would not approach the patient for exercise until a transfusion was complete. If a patient's platelets were 10-20 bil/L, resistance exercise would only include resistance tubing rather than dumbbells to avoid increased risk of bruising and bleeding. These guidelines were based on prior trials in this setting $[19,20]$ and discussions with our leukemia physicians.

\subsection{Exercise feasibility, safety, and adherence}

We assessed feasibility by examining recruitment rate (\% of eligible patients recruited) and retention rate (\% of consented patients who completed the intervention). Safety was assessed daily throughout the intervention. All exercise sessions were monitored by the CEP and any adverse events were captured using the National Cancer Institute: Cancer Therapy Evaluation Program Common Terminology Criteria version 3.0. Adherence was monitored by the CEP through weekly tracking sheets and daily exercise logs. Reasons for nonparticipation on scheduled exercise days and any bouts of unsupervised exercise were also documented using these forms. Two components of adherence were examined: the percentage of supervised exercise days (the ratio of supervised exercise to days approached for supervised exercise) and the number of weeks in which participants met Canada's Physical Activity Guidelines [24]. 


\subsection{Outcome assessments}

Patients completed assessments at the following time points: (1) at baseline (within 7 days of starting IC); (2) post-induction (within 1 week of discharge after completing IC); and (3) post cycle 2 of chemotherapy (4-6 weeks following discharge after induction). At each assessment, all fitness measures and patient-reported outcomes were completed (see below). At baseline, pre-symptom exercise levels were captured with the Godin Leisure Time Exercise Questionnaire [25]. Remission status was also collected at time points 2 and 3. In the present analysis of intervention efficacy, we focus on changes from baseline to postinduction only.

\subsection{Fitness evaluation}

Anthropometric measures (body mass index) and basic physiological parameters (resting heart rate, blood pressure, oxygen saturation) were collected. Aerobic fitness $\left(\mathrm{VO}_{2}\right.$ peak) was assessed using a validated treadmill maximal exercise test, the modified Bruce protocol [23]. The modified Bruce treadmill test is a walking-based continuous protocol. The test is comprised of 3 min workloads. The speed and/or intensity increases with each workload. We monitored heart rate and RPE throughout the test. The primary criteria for test termination were: (a) achievement of a heart rate within 10-15 beats per minute of agepredicted maximum heart rate or (b) reported RPE of $>/=9$. Secondary criteria for test termination included: (a) patient request to stop and (b) symptoms including lightheadedness, dizziness, etc. The speed and incline achieved during the final workload of the test were used to derive a $\mathrm{VO}_{2}$ peak value using the ACSM walking equation [23]. $\mathrm{The} \mathrm{VO}_{2}$ peak value calculated from the ACSM walking equation (in $\mathrm{ml} \mathrm{kg}^{-1} \mathrm{~min}^{-1}$ ) was converted to METS for reporting purposes by dividing the $\mathrm{VO}_{2}$ peak (in $\mathrm{ml} \mathrm{kg}^{-1} \mathrm{~min}^{-1}$ ) by 3.5. A CEP conducted all aerobic fitness testing. A physician was not present during testing. Patients were permitted to hold the handrails of the treadmill throughout the test. We chose to allow patients to do so in order to minimize the risk for falls, particularly because of the increased risk of bleeding due to low platelet counts. Functional endurance was evaluated using the 6 min walk test (6MWT) [26]. Grip strength [27], as a measure of upper body strength, was assessed with a Jamar dynamometer (Sammons-Preston, IL, USA) [28]. Lower body function was evaluated using the timed 10-chair stand test [29].

\subsection{Patient-reported outcomes}

QOL was measured with the European Organisation for the Research and Treatment of Cancer (EORTC) core 30-item questionnaire (QLQ-C30). The EORTC QLQ-C30 is a widely used, self-reported, multi-dimensional, psychometrically sound cancer QOL instrument [30]. It features a global QOL measure, 5 functional scales (physical, role, cognitive, emotional, and social) and various symptom items. Fatigue was assessed using the functional assessment of cancer therapy fatigue subscale (FACT-Fatigue). The FACTFatigue consists of 13 questions and has high test-retest reliability $(r=0.87)$, excellent internal consistency (Cronbach's $a=0.93-0.95$ ), and good validity [31,32]. A global singleitem fatigue visual analog scale was also obtained using the Edmonton Symptom Assessment Scale [33]. Depression and anxiety were measured using the 14-item Hospital Anxiety and Depression Scale (HADS) [34], which has been validated in cancer patients [35].

\subsection{AML treatment tolerability}

Based on theoretical considerations and prior literature, we defined four outcomes to reflect treatment tolerability that may be modified by physical fitness. At discharge we captured length of stay in hospital, occurrence of sepsis [36], and ICU admission. We also captured time to start cycle 2 of chemotherapy. 


\subsection{Statistical analyses}

Baseline characteristics were described with means for continuous variables and counts for categorical variables. Patients were stratified a priori into under 60 and 60 or older.

2.9.1. Primary objectives-To assess feasibility of recruitment and retention, descriptive analyses of the recruitment log was performed. Reasons for non-participation were categorized. Descriptive analyses of adherence were also performed. Reasons why exercise could not be performed on other days were categorized and tallied.

To provide estimates of the effect of exercise on fitness outcomes, multivariable linear regression models [37] were constructed using change scores for each of the fitness measures. Age, gender, adherence, and baseline fitness level were included as covariates in these models. We utilized a $p$-value of 0.05 for all comparisons.

2.9.2. Secondary objectives-To provide estimates of the effect of exercise on QOL and fatigue, we again constructed adjusted linear regression models using both the QLQC30 global QOL and FACT-F change scores as outcome measures. Given the small sample size and the fact that patient-reported outcomes were secondary endpoints, formal statistical comparisons were not done by age group.

The impact of exercise on AML treatment tolerability was examined with descriptive statistics. To examine safety, we reported the frequency of significant clinical adverse events that occurred within $24 \mathrm{~h}$ of exercise.

\section{Results}

\subsection{Baseline characteristics}

Baseline characteristics, stratified by age, are presented in Table 1. The mean age of all participants was 56.4 years. No significant comorbidity was identified in either group. The majority $(51.4 \%)$ of participants had normal cytogenetics. Hemoglobin levels at baseline were significantly different between the two age groups; those $60+$ years presented with a lower hemoglobin value at baseline $(83.1 \mathrm{~g} / \mathrm{L}$ vs. $89.9 \mathrm{~g} / \mathrm{L} ; p=0.028)$. There were no significant differences between those $<60$ and $60+$ years in any physical measures at baseline. However, results of all physical measures were lower than values observed in a healthy population $[23,28]$. There were no significant differences between those $<60$ years and 60+ years in any of the QOL measures at baseline (Table 1).

\subsection{Feasibility and adherence}

Among all eligible participants ( $n=52), 35$ consented to participate (recruitment rate 67\%). Retention was excellent (97\%) with only one premature dropout following the baseline assessment (Fig. 1).

Adherence to the exercise intervention (number of days of supervised exercise performed) and compliance with the Canadian Physical Activity Guidelines are reported in Table 2. Mean adherence in terms of number of days of supervised exercise was 8.1 days (range 017), and was similar in the two age groups. Adherence to Canadian guidelines was low at $5.9 \%$, and was also similar by age group (Table 2 ).

Throughout the intervention, daily reasons for not exercising were documented and categorized. The most commonly reported reason for not participating in daily exercise was fatigue. Additional reasons are listed in Table 3. 


\subsection{Objective physical measures}

Functional endurance, assessed using the $6 \mathrm{MWT}$, was significantly better post-induction compared to baseline (mean difference $213.7 \mathrm{ft}$., $p=0.006$ ). Improvements were also observed in aerobic fitness $\left(\mathrm{VO}_{2}\right.$ peak, mean difference 0.5 METS $)$ and chair stand performance (mean improvement $1.9 \mathrm{~s}$ ), although neither was statistically significant. Grip strength declined significantly over the intervention course (mean decrease $2.3 \mathrm{~kg}, p=$ 0.009) (Table 4). Although numerical improvements in $6 \mathrm{MWT}$ and $\mathrm{VO}_{2}$ peak were similar by age group, older adults appeared not to improve in chair stand performance over time and had greater declines in grip strength compared to younger adults (Table 4).

\subsection{Patient-reported measures}

There were no statistically significant changes in global QOL or FACT-F measures, although both did exhibit trends toward improvement from baseline. Clinically significant improvements (3-point change) in fatigue were observed in the $<60$ group. There was a significant decline in anxiety from baseline among all participants $(p=0.03)$. Among QOL domains, emotional function appeared to improve the most, although this was not statistically significant $(p=0.08)$ (Table 5).

\subsection{Treatment tolerability}

The mean length of stay for all participants was 37.2 (SD 10.6) days. Seventy-one percent of all participants achieved complete remission following induction. Remission rates were higher in those $<60$ years $(77.8 \%)$ compared to those $60+$ years $(62.5 \%), p=0.22$.

Nearly half (43.8\%) of participants in the $60+$ group developed sepsis during their in-patient admission compared to $11.1 \%$ of those $<60$ years $(p=0.052)$. Although not statistically significant $(p=0.60)$, those $<60$ years were more frequently admitted to the ICU $(16.7 \%)$ than their older counterparts (6.3\%). Time to cycle 2 of chemotherapy both from the start of induction (cycle 1) and from discharge at the end of induction were 49.9 (SD 10.0) days and 16.8 (SD 5.6) days, respectively (Table 6).

\subsection{Safety}

In over 600 patient-days of observation, one possible grade II musculoskeletal event occurred (neck/back pain limiting function for $24 \mathrm{~h}$ ). No other adverse events or safety concerns were reported.

\section{Discussion}

We studied the feasibility, safety, and preliminary efficacy of a supervised exercise program during IC in 35 adult patients in this non-randomized clinical trial. We were able to demonstrate high rates of recruitment, retention, and excellent safety of the intervention as well as a statistically significant and clinically important improvement in 6MWT distance. Although not all were statistically significant, potentially clinically important improvements in several patient-reported outcomes were also observed (anxiety, fatigue, global QOL, and emotional function).

Although four prior exercise studies have been done in patients with AML undergoing IC, significant differences in the exercise regimens and measured outcomes make it difficult to draw definitive conclusions about the value of exercise in this setting. While these studies have demonstrated improvements in a variety of fitness and QOL outcomes, findings have been inconsistent and study samples have been small. Moreover, the impact of exercise on AML treatment toxicity, a clinically important outcome, was only reported in one study for 
one outcome (length of stay) [20]. More definitive trials are needed prior to adoption into clinical practice.

Our results are tempered by significant challenges in administering a daily exercise program to a relatively unwell population. Despite having a dedicated exercise specialist approaching patients multiple times per day five days a week, as well as a supportive clinical nursing and medical team, our greatest challenge was fairly low adherence rates. Most commonly, patients were not feeling well enough to exercise on a given day either due to fatigue, fever, generally feeling unwell, or nausea. These factors are not, in our experience, easy to correct with improved supportive care, and innovative approaches may be required to facilitate greater patient adherence. Of the four prior AML exercise studies, only Klepin et al. reported adherence measures, including the percentage of participants who attended at least one exercise session (70.8\%) and the mean number of exercise sessions attended (2.7) [21]. Although our adherence rates are better than those reported by Klepin et al. $(94.1 \%$ and 8.1 sessions, respectively), we approached patients more often each week and included both younger and older patients, whereas Klepin et al. included only patients over the age of 55 .

An additional observation relates to somewhat unanticipated declines in grip strength over time among study participants. This is most likely due to the profound malnutrition and weight loss that occur in these patients as a consequence of mucositis, nausea, and other chemotherapy-related toxicities. On average our patients lost about $4 \mathrm{~kg}$ (almost 5\%) of body weight. Such a significant loss of body weight in a short period of time would include a significant loss of muscle mass, attenuating many of the benefits of exercise. In cancer patients with cachexia, successful exercise programs typically include a resistance component along with an aerobic component [15]. Thus, any successful future exercise intervention during treatment should include resistance exercises and consider a parallel nutritional support intervention. Additionally, grip strength has not been validated as a measure of total body muscle strength in oncology patients receiving high-dose chemotherapy. It may be useful to include additional measures of upper body strength in future trials.

Our results have confirmed the feasibility and safety of a carefully designed and controlled intervention in both older and younger AML patients. Patients undergoing IC are interested in and are able to participate in light to moderate intensity exercise during in-patient admission for curative treatment. However, without a proper control group, comments on efficacy of fitness and QOL endpoints must be cautiously interpreted. These results provide a base from which we can design and implement future interventions. We are currently enrolling patients in a randomized phase II study to more precisely characterize efficacy outcomes and provide additional data to help inform a multicenter phase III RCT.

In addition to furthering our understanding of exercise in this patient population through the randomized trial that we are currently completing and ultimately through a phase III multicenter trial, additional research in this area would benefit from a focus on treatment toxicity. In this trial, we systematically collected data on four possible treatment toxicity outcomes that may be positively affected by exercise. Although these data are difficult to interpret in the absence of either historical data or a control group, they provide a baseline for our future studies and, we hope, will stimulate other investigators to collect similar outcomes.

In addition to investigating treatment toxicity, future work in this area should investigate the role of nutrition during IC and nutrition support that may work in conjunction with exercise as a means to maintain physical function and QOL throughout the course of treatment. 


\section{Acknowledgments}

We would like to thank the participants, nurses, and doctors of the Leukemia Program, Department of Medical Oncology and Hematology, Princess Margaret Hospital. Dr. Alibhai is a Research Scientist of the Canadian Cancer Society.

Role of the funding source. This study was supported by a grant from the Toronto Rehabilitation Institute and a donation from Toronto Dominion Securities Inc. Study sponsors had no role in the study design, collection, analysis and interpretation of data or in the writing of this manuscript or the decision to submit for publication.

\section{References}

1. Sekeres MA, Stone RM. The challenge of acute myeloid leukemia in older patients. Curr Opin Oncol. 2002; 14:24-30. [PubMed: 11790976]

2. Stein RS, Vogler WR, Winton EF, Cohen HJ, Raney MR, Bartolucci A. Therapy of acute myelogenous leukemia in patients over the age of 50: a randomized Southeastern Cancer Study Group trial. Leuk Res. 1990; 14:895-903. [PubMed: 2259226]

3. Taylor PR, Reid MM, Stark AN, Bown N, Hamilton PJ, Proctor SJ. De novo acute myeloid leukaemia in patients over 55-years-old: a population-based study of incidence, treatment and outcome. Northern Region Haematology Group. Leukemia. 1995; 9:231-7. [PubMed: 7532766]

4. Stalfelt AM. Quality of life of patients with acute myeloid leukaemia. Leuk Res. 1994; 18:257-67. [PubMed: 8170170]

5. Schumacher A, Kessler T, Buchner T, Wewers D, van de Loo J. Quality of life in adult patients with acute myeloid leukemia receiving intensive and prolonged chemotherapy - a longitudinal study. Leukemia. 1998; 12:586-92. [PubMed: 9557618]

6. Schumacher A, Wewers D, Heinecke A, Sauerland C, Koch OM, van de Loo J, et al. Fatigue as an important aspect of quality of life in patients with acute myeloid leukemia. Leuk Res. 2002; 26:355-62. [PubMed: 11839378]

7. Alibhai SM, Leach M, Kowgier ME, Tomlinson GA, Brandwein JM, Minden MD. Fatigue in older adults with acute myeloid leukemia: predictors and associations with quality of life and functional status. Leukemia. 2007; 21:845-8. [PubMed: 17287855]

8. Sekeres MA, Stone RM, Zahrieh D, Neuberg D, Morrison V, De Angelo DJ, et al. Decision-making and quality of life in older adults with acute myeloid leukemia or advanced myelodysplastic syndrome. Leukemia. 2004; 18:809-16. [PubMed: 14762444]

9. Schmitz KH, Holtzman J, Courneya KS, Masse LC, Duval S, Kane R. Controlled physical activity trials in cancer survivors: a systematic review and meta-analysis. Cancer Epidemiol Biomarkers Prev. 2005; 14:1588-95. [PubMed: 16030088]

10. Conn VS, Hafdahl AR, Porock DC, McDaniel R, Nielsen PJ. A meta-analysis of exercise interventions among people treated for cancer. Support Care Cancer. 2006; 14:699-712. [PubMed: 16447036]

11. Sauter E, Daly MB. Physical activity across the cancer continuum: report of a workshop: review of existing knowledge and innovative designs for future research. Cancer. 2002; 95:1134-43. [PubMed: 12209701]

12. Schneider CM, Hsieh CC, Sprod LK, Carter SD, Hayward R. Cancer treatment-induced alterations in muscular fitness and quality of life: the role of exercise training. Ann Oncol. 2007; 18:1957-62. [PubMed: 17804476]

13. Liu RD, Chinapaw MJ, Huijgens PC, van Mechelen W. Physical exercise interventions in haematological cancer patients, feasible to conduct but effectiveness to be established: a systematic literature review. Cancer Treat Rev. 2009; 35:185-92. [PubMed: 19004560]

14. Watson T, Mock V. Exercise as an intervention for cancer-related fatigue. Phys Ther. 2004; 84:736-43. [PubMed: 15283624]

15. Schmitz KH, Courneya KS, Matthews C, Demark-Wahnefried W, Galvao DA, Pinto BM, et al. American College of Sports Medicine roundtable on exercise guidelines for cancer survivors. Med Sci Sports Exerc. 2010; 42:1409-26. [PubMed: 20559064] 
16. McNeely ML, Campbell KL, Rowe BH, Klassen TP, Mackey JR, Courneya KS. Effects of exercise on breast cancer patients and survivors: a systematic review and meta-analysis. CMAJ. 2006; 175:34-41. [PubMed: 16818906]

17. Thorsen L, Courneya KS, Stevinson C, Fossa SD. A systematic review of physical activity in prostate cancer survivors: outcomes, prevalence, and determinants. Support Care Cancer. 2008; 16:987-97. [PubMed: 18274783]

18. Chang PH, Lai YH, Shun SC, Lin LY, Chen ML, Yang Y, et al. Effects of a walking intervention on fatigue-related experiences of hospitalized acute myelogenous leukemia patients undergoing chemotherapy: a randomized controlled trial. J Pain Symptom Manage. 2008; 35:524-34. [PubMed: 18280104]

19. Battaglini CL, Hackney AC, Garcia R, Groff D, Evans E, Shea T. The effects of an exercise program in leukemia patients. Integr Cancer Ther. 2009; 8:130-8. [PubMed: 19679621]

20. de Wit M, Seyfi Z, Susse Y, Oechsle K, Bokemeyer C. Effects of aerobic exercise training on physical performance during myeloablative therapy [abstract]. J Clin Oncol. 2008; 26:502S.

21. Klepin HD, Danhauer SC, Tooze JA, Stott K, Daley K, Vishnevsky T, et al. Exercise for older adult inpatients with acute myelogenous leukemia: a pilot study. J Geriatr Oncol. 2011; 2:11-7. [PubMed: 23843929]

22. Hayes SC, Spence RR, Galvao DA, Newton RU. Australian Association for Exercise and Sport Science position stand: optimising cancer outcomes through exercise. J Sci Med Sport. 2009; 12:428-34. [PubMed: 19428291]

23. Heyward, VH. Advanced Fitness Assessment and Exercise Prescription. 5. Human Kinetics Publishers; Windsor: 2006.

24. Canadian Society of Exercise Physiology. Canadian Physical Activity Guidelines. Ottawa: Health Canada; 2011.

25. Godin G, Shephard RJ. A simple method to assess exercise behavior in the community. Can J Appl Sport Sci. 1985; 10:141-6. [PubMed: 4053261]

26. Enright PL, McBurnie MA, Bittner V, Tracy RP, McNamara R, Arnold A, et al. The 6 min walk test: a quick measure of functional status in elderly adults. Chest. 2003; 123:387-98. [PubMed: 12576356]

27. Rantanen T, Volpato S, Ferrucci L, Heikkinen E, Fried LP, Guralnik JM. Handgrip strength and cause-specific and total mortality in older disabled women: exploring the mechanism. J Am Geriatr Soc. 2003; 51:636-41. [PubMed: 12752838]

28. Mathiowetz V, Kashman N, Volland G, Weber K, Dowe M, Rogers S. Grip and pinch strength: normative data for adults. Arch Phys Med Rehabil. 1985; 66:69-74. [PubMed: 3970660]

29. Curb JD, Ceria-Ulep CD, Rodriguez BL, Grove J, Guralnik J, Willcox BJ, et al. Performancebased measures of physical function for high-function populations. J Am Geriatr Soc. 2006; 54:737-42. [PubMed: 16696737]

30. Aaronson NK, Ahmedzai S, Bergman B, Bullinger M, Cull A, Duez NJ, et al. The European Organization for Research and Treatment of Cancer QLQ-C30: a quality-of-life instrument for use in international clinical trials in oncology. J Natl Cancer Inst. 1993; 85:365-76. [PubMed: 8433390]

31. Yellen SB, Cella DF, Webster K, Blendowski C, Kaplan E. Measuring fatigue and other anemiarelated symptoms with the functional assessment of cancer therapy (FACT) measurement system. J Pain Symptom Manage. 1997; 13:63-74. [PubMed: 9095563]

32. Cella D. The functional assessment of cancer therapy-anemia (FACT-An) Scale: a new tool for the assessment of outcomes in cancer anemia and fatigue. Semin Hematol. 1997; 34:13-9. [PubMed: 9253779]

33. Bruera E, Kuehn N, Miller MJ, Selmser P, Macmillan K. The edmonton symptom assessment system (ESAS): a simple method for the assessment of palliative care patients. J Palliat Care. 1991; 7:6-9. [PubMed: 1714502]

34. Zigmond AS, Snaith RP. The hospital anxiety and depression scale. Acta Psychiatr Scand. 1983; 67:361-70. [PubMed: 6880820] 
35. Walker J, Postma K, McHugh GS, Rush R, Coyle B, Strong V, et al. Performance of the hospital anxiety and depression scale as a screening tool for major depressive disorder in cancer patients. $\mathrm{J}$ Psychosom Res. 2007; 63:83-91. [PubMed: 17586341]

36. Bone RC, Balk RA, Cerra FB, Dellinger RP, Fein AM, Knaus WA, et al. Definitions for sepsis and organ failure and guidelines for the use of innovative therapies in sepsis. The ACCP/SCCM Consensus Conference Committee. American College of Chest Physicians/Society of Critical Care. Medicine Chest. 1992; 101:1644-55.

37. Diggle, PJ.; Liang, K-Y.; Zeger, SL. Analysis of Longitudinal Data. New York: Oxford University Press; 1994. 
Patients initiating IC for de novo or relapsed AML at PMH assessed for eligibility $(n=78)$

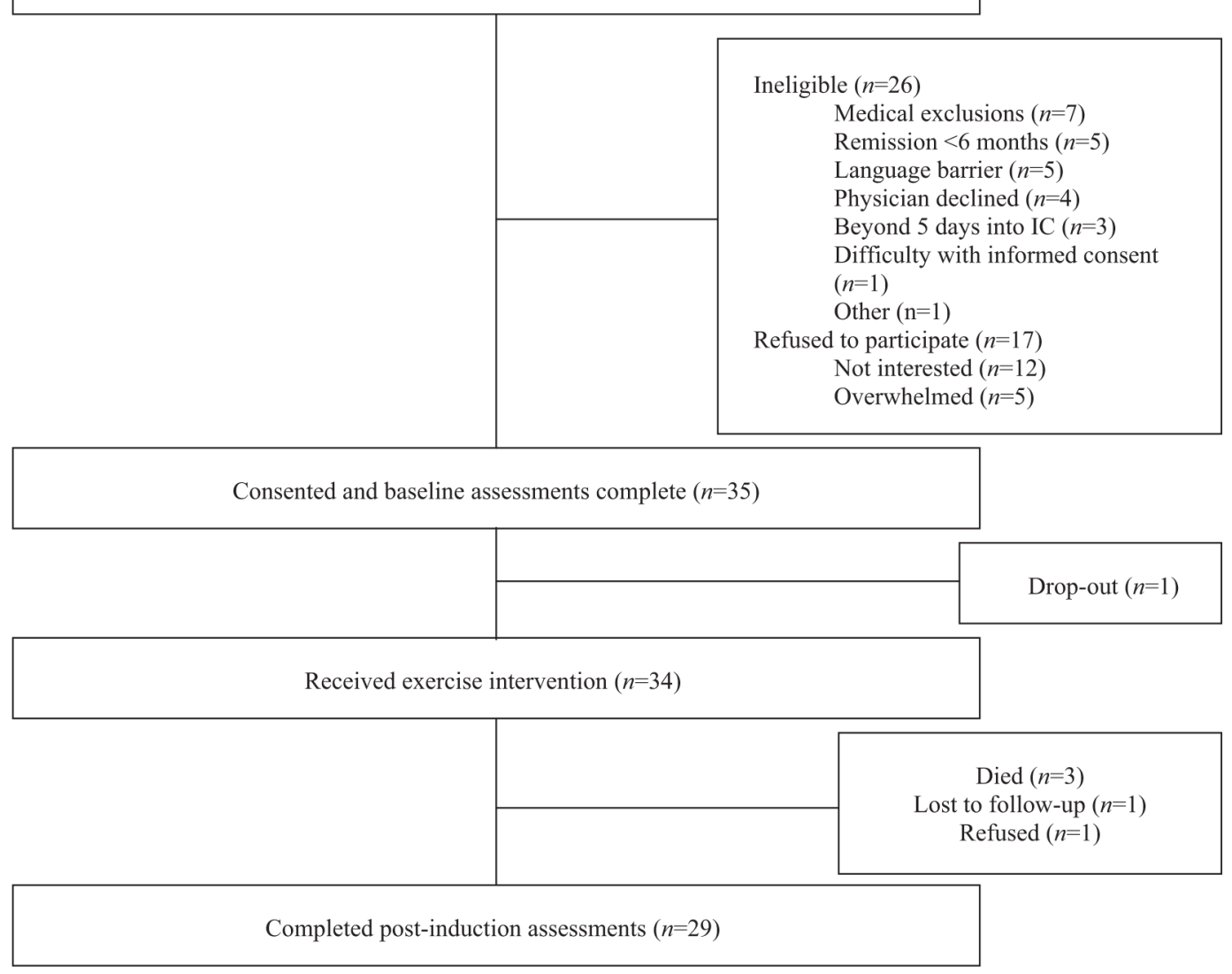

Fig. 1.

Participant flow, CONSORT flow diagram showing patient recruitment and retention. 
Table 1

Baseline patient demographics, disease characteristics, physical fitness and self-report measures stratified by age.

\begin{tabular}{|c|c|c|c|c|}
\hline \multirow[t]{2}{*}{ Variable } & \multicolumn{3}{|c|}{ Mean (SD) or percent } & \multirow[t]{2}{*}{$p$} \\
\hline & All $(n=35)$ & $<60$ y $(n=18)$ & 60 y $(n=17)$ & \\
\hline \multicolumn{5}{|l|}{ Demographics } \\
\hline Age (years) & $56.4(12.9)$ & $46.6(9.6)$ & $66.8(6.0)$ & 0.001 \\
\hline Gender ( $\%$ female) & 54.3 & 66.7 & 41.1 & 0.18 \\
\hline Race (\% white) & 74.3 & 66.7 & 82.4 & 0.44 \\
\hline Marital status (\% married) & 77.1 & 72.2 & 82.4 & 0.69 \\
\hline Education ( $\%$ beyond high school education) & 57.1 & 55.6 & 58.8 & 0.74 \\
\hline Working status (\% retired) & 42.9 & 16.7 & 70.6 & 0.01 \\
\hline \multicolumn{5}{|l|}{ Clinical characteristics } \\
\hline Weight (kg) & $74.3(18.2)$ & $76.1(19.8)$ & $72.3(16.6)$ & 0.50 \\
\hline BMI $\left(\mathrm{kg} / \mathrm{m}^{2}\right)$ & $26.6(5.7)$ & $27.7(6.7)$ & $25.5(4.5)$ & 0.26 \\
\hline Charlson score (median) & 0 & 0 & 0 & 0.77 \\
\hline ECOG Performance Status (median) & 1 & 1 & 1 & 1.00 \\
\hline Karnofsky score (median) & 80 & 80 & 80 & 0.86 \\
\hline Cytogenetics & & & & 0.42 \\
\hline Normal cytogenetics & $51.4 \%$ & $44.4 \%$ & $58.8 \%$ & \\
\hline Favorable & $8.6 \%$ & $11.1 \%$ & $5.9 \%$ & \\
\hline Intermediate & $8.6 \%$ & $11.1 \%$ & $5.9 \%$ & \\
\hline Unfavorable & $17.1 \%$ & $16.7 \%$ & $17.6 \%$ & \\
\hline Inconclusive/failed & $14.3 \%$ & $16.7 \%$ & $11.8 \%$ & \\
\hline \multicolumn{5}{|l|}{ Molecular studies $b$} \\
\hline NPM1 positive & $14.3 \%$ & $27.8 \%$ & $0 \%$ & $0.12 \%$ \\
\hline FLT3 positive & $17.1 \%$ & $33.3 \%$ & $0 \%$ & $0.11 \%$ \\
\hline Hemoglobin $(g / L)$ & $86.6(9.3)$ & $89.9(10.7)$ & $83.1(6.1)$ & 0.03 \\
\hline White blood cell counts $\left(\mathrm{X} 10^{9} / \mathrm{L}\right)$ & $6.5(21.8)$ & $3.4(7.9)$ & $9.4(30.4)$ & 0.45 \\
\hline Platelets $\left(\mathrm{X} 10^{9} / \mathrm{L}\right)$ & $55.2(56.1)$ & $55.3(52.4)$ & $55.1(61.5)$ & 0.99 \\
\hline \multicolumn{5}{|l|}{ Objective physical function } \\
\hline $\mathrm{VO}_{2}$ peak (METS) & $6.9(1.8)$ & $7.5(1.8)$ & $6.2(1.6)$ & 0.16 \\
\hline 6-MWT (feet) & $962.5(286.3)$ & $1031.3(203.4)$ & $893.8(343.8)$ & 0.18 \\
\hline Chair stands (seconds) & $33.0(26.0)$ & $36.5(34.3)$ & $29.4(12.6)$ & 0.44 \\
\hline Grip strength (kg) & $23.9(11.0)$ & $24.9(11.4)$ & $22.9(10.9)$ & 0.60 \\
\hline \multicolumn{5}{|l|}{ Self-report measures ${ }^{a}$} \\
\hline Global QOL (range 0-100) & $45.1(26.6)$ & $42.7(25.4)$ & $47.4(28.3)$ & 0.63 \\
\hline Physical Functioning Scale (range 0-100) & $77.2(23.3)$ & $76.3(21.4)$ & $78.2(25.9)$ & 0.82 \\
\hline Role Functioning Scale (range 0-100) & $44.8(33.5)$ & $43.8(32.1)$ & $45.8(35.7)$ & 0.86 \\
\hline Emotional Functioning Scale (range 0-100) & $70.6(26.0)$ & $65.1(27.1)$ & $76.0(24.5)$ & 0.24 \\
\hline Cognitive Functioning Scale (range 0-100) & $76.9(25.3)$ & $73.9(23.5)$ & 80.0 (27.6) & 0.52 \\
\hline Social Functioning Scale (range 0-100) & $47.3(35.5)$ & $45.6(33.6)$ & $48.9(38.2)$ & 0.79 \\
\hline
\end{tabular}




\begin{tabular}{|c|c|c|c|c|}
\hline \multirow[t]{2}{*}{ Variable } & \multicolumn{3}{|c|}{ Mean (SD) or percent } & \multirow[t]{2}{*}{$p$} \\
\hline & All $(n=35)$ & $<60$ y $(n=18)$ & $\lcm{60} \mathrm{y}(n=17)$ & \\
\hline Fatigue VAS (range 0-10) & $4.6(2.6)$ & $4.0(2.3)$ & $5.2(2.8)$ & 0.19 \\
\hline FACT-F (range 0-52) & $31.5(13.1)$ & $32.3(12.5)$ & $30.8(14.0)$ & 0.76 \\
\hline HADS-Anxiety (range 0-21) & $6.2(4.7)$ & $7.4(4.6)$ & $4.9(4.6)$ & 0.16 \\
\hline HADS-Depression (range 0-21) & $5.9(4.1)$ & $6.1(4.3)$ & $5.6(4.0)$ & 0.73 \\
\hline Godin LTEQ (MET-hours per week MVPA) & $10.8(14.6)$ & $8.6(11.1)$ & $13.3(17.8)$ & 0.35 \\
\hline
\end{tabular}

Values are means unless indicated otherwise.

aHigher scores on all self-report measures indicate better quality of life and fewer symptoms, other than the HADS and the Fatigue VAS.

$\mathrm{SD}=$ Standard deviation; $\mathrm{BMI}=$ Body mass index ECOG $=$ Eastern cooperative oncology group; 6-MWT $=6$ min walk test; $\mathrm{QOL}=\mathrm{Quality}$ of life; FACT-F $=$ Functional assessment of cancer therapy - Fatigue; HADS $=$ Hospital anxiety and depression scale; VAS = Visual analog scale; METS = Metabolic equivalents; LTEQ = Leisure-time exercise questionnaire; MVPA = moderate-vigorous physical activity.

${ }^{b}$ Molecular studies were only done in cytogenetically normal patients 
Table 2

Program adherence stratified by age.

\begin{tabular}{|c|c|c|c|}
\hline & All $(n=34)$ & $<60 y(n=18)$ & $\searrow 60 y(n=16)$ \\
\hline Supervised exercise (days), mean (range) & $8.1(0-17)$ & $7.9(0-17)$ & $8.4(0-15)$ \\
\hline Ratio of supervised exercise: days approached & $45.8 \%$ & $46.9 \%$ & $44.6 \%$ \\
\hline Adherence to Canada's PA Guidelines: & - & - & - \\
\hline Throughout admission & $5.9 \%$ & $5.6 \%$ & $6.3 \%$ \\
\hline One or more weeks during admission & $29.4 \%$ & $22.2 \%$ & $37.5 \%$ \\
\hline
\end{tabular}

PA $=$ Physical activity 


\section{Table 3}

Patient-reported reasons for declining exercise on a given day when approached that day.

\begin{tabular}{lr}
\hline Reported reasons & \% \\
\hline Fatigue & 33.5 \\
Medical exclusion & 14.9 \\
Unavailable/sleeping & 9.5 \\
Fever/general unwell & 8.9 \\
Nausea/vomiting & 6.7 \\
Bleeding & 4.1 \\
Pain & 3.9 \\
Delay in transfusion & 3.6 \\
Transfusion related symptoms & 3.6 \\
Hypotension & 2.2 \\
Diarrhea & 1.2 \\
Dizziness & 1.2 \\
Weakness & 1.4 \\
Headache & 0.5 \\
Other & 4.8 \\
\hline
\end{tabular}

This table enumerates reasons participants provided on a given day if they declined exercise when approached that day. Patients may have provided more than one reason on a given day. 
Table 5

Change in self-report measures from baseline to post-induction.

\begin{tabular}{lccrl}
\hline Variable & Baseline & Post-induction & Change $^{\boldsymbol{a}}$ & $\boldsymbol{p}$-value \\
\hline Global QOL & $45.1(26.6)$ & $51.4(21.4)$ & 6.3 & 0.28 \\
Physical functioning & $77.2(23.3)$ & $73.4(24.9)$ & -3.8 & 0.50 \\
Role functioning & $44.8(33.5)$ & $41.5(30.8)$ & -3.3 & 0.71 \\
Emotional functioning & $70.6(26.0)$ & $79.6(20.9)$ & 9.0 & 0.08 \\
Cognitive functioning & $76.9(25.3)$ & $74.1(27.9)$ & -2.8 & 0.59 \\
Social functioning & $47.3(35.5)$ & $50.8(29.6)$ & 3.5 & 0.66 \\
FACT-F & $31.5(13.1)$ & $33.9(11.7)$ & 2.4 & 0.34 \\
Fatigue VAS & $4.6(2.6)$ & $4.7(2.6)$ & 0.1 & 0.91 \\
HADS anxiety & $6.2(4.7)$ & $4.3(4.4)$ & -1.9 & 0.03 \\
HADS depression & $5.9(4.1)$ & $4.7(3.8)$ & -0.8 & 0.25 \\
\hline
\end{tabular}

Self-report measures are reported as mean (standard deviation) scores for all participants as there were no significant differences between age groups. QOL = Quality of life; FACT-F = Functional assessment of cancer therapy - Fatigue subscale; VAS = Visual analog scale; HADS = Hospital anxiety and depression scale.

${ }^{a}$ Minimum clinically important differences are 10 points for QOL domains, 3 points for the FACT-F, 1.5 points for the Fatigue VAS, and 2 points for the HADS subscales. 
Table 6

Treatment tolerability stratified by age.

\begin{tabular}{lllcl}
\hline & All $(\boldsymbol{n = 3 4 )}$ & $<\mathbf{6 0} \mathbf{y}(\boldsymbol{n}=\mathbf{1 8})$ & $\mathbf{7 6 0} \mathbf{y}(\boldsymbol{n}=\mathbf{1 6})$ & $\boldsymbol{p}$ \\
\hline Length of stay, days, Mean(SD) & $37.2(10.6)$ & $34.2(9.4)$ & $40.5(11.2)$ & 0.09 \\
Occurrence of sepsis & $26.5 \%$ & $11.1 \%$ & $43.8 \%$ & 0.052 \\
ICU admission & $11.5 \%$ & $16.7 \%$ & $6.3 \%$ & 0.60 \\
Complete remission post IC & $70.6 \%$ & $77.8 \%$ & $62.5 \%$ & 0.22 \\
Time to cycle 2 of chemotherapy from the start of IC, mean (SD) number of days & $49.9(10.0)$ & $48.0(11.5)$ & $53.0(6.4)$ & 0.32 \\
Time to cycle 2 of chemotherapy from discharge, mean (SD) number of days & $16.8(5.6)$ & $14.5(4.7)$ & $20.7(5.2)$ & 0.015 \\
\hline
\end{tabular}

$\mathrm{ICU}=$ Intensive care unit; IC = Induction chemotherapy. 